\author{
ECONOMIC GROWTH CENTER \\ YALE UNIVERSITY \\ P.O. Box 208629 \\ New Haven, CT 06520-8269 \\ http://www.econ.yale.edu/ egcenter/
}

\title{
Two Algorithms for Solving the Walrasian Equilibrium Inequalities
}

\author{
Donald J. Brown \\ Yale University \\ Ravi Kannan \\ Yale University
}

October 2006

Notes: Center Discussion Papers are preliminary materials circulated to stimulate discussions and critical comments.

This paper can be downloaded without charge from the Social Science Research Network electronic library at: http://ssrn.com/abstract=940093

An index to papers in the Economic Growth Center Discussion Paper Series is located at: http://www.econ.yale.edu/ egcenter/research.htm 


\title{
Two Algorithms for Solving the Walrasian Equilibrium Inequalities
}

Donald J. Brown and Ravi Kannan

\begin{abstract}
We propose two algorithms for deciding if the Walrasian equilibrium inequalities are solvable. These algorithms may serve as nonparametric tests for multiple calibration of applied general equilibrium models or they can be used to compute counterfactual equilibria in applied general equilibrium models defined by the Walrasian equilibrium inequalities.
\end{abstract}

Keywords: Applied general equilibrium analysis, Walrasian equilibrium inequalities, calibration

JEL Codes C63, C68, D51, D58 


\title{
Two Algorithms for Solving the Walrasian Equilibrium Inequalities
}

\author{
Donald J. Brown* Ravi Kannan ${ }^{\dagger}$
}

\begin{abstract}
We propose two algorithms for deciding if the Walrasian equilibrium inequalities are solvable. These algorithms may serve as nonparametric tests for multiple calibration of applied general equilibrium models or they can be used to compute counterfactual equilibria in applied general equilibrium models defined by the Walrasian equilibrium inequalities.

Keywords: Applied general equilibrium analysis, Walrasian equilibrium inequalities, Calibration

JEL Classification: C63, C68, D51, D58
\end{abstract}

\section{Introduction}

Numerical specifications of applied microeconomic general equilibrium models are inherently indeterminate. Simply put, there are more unknowns (parameters) than equations (general equilibrium restrictions). Calibration of parameterized numerical general equilibrium models resolves this indeterminacy using market data from a "benchmark year"; parameter values gleaned from the empirical literature on production functions and demand functions; and the general equilibrium restrictions. The calibrated model allows the simulation and evaluation of alternative policy prescriptions, such as changes in the tax structure, by using Scarf's algorithm or one of its variants to compute counterfactual equilibria. Not surprisingly, the legitimacy of calibration as a methodology for specifying numerical general equilibrium models is the subject of an ongoing debate within the profession, ably surveyed by Dawkins et al. (2002). In their survey, they briefly discuss multiple calibration. That is, choosing parameter values for numerical general equilibrium models consistent with market data for two or more years. It is the implications of this notion that we explore in this paper.

Our approach to counterfactual analysis derives from Varian's unique insight that nonparametric analysis of demand or production data admits extrapolation, i.e.,

\footnotetext{
${ }^{*}$ Economic Growth Center and Cowles Foundation, Yale University

${ }^{\dagger}$ Department of Computer Science, Yale University
} 
"given observed behavior in some economic environments, we can forecast behavior in other environments", Varian $(1982,1984)$. The forecast behavior in applied general equilibrium analysis is the set of counterfactual equilibria.

Here is an example inspired by the discussion of extrapolation in Varian (1982), illustrating the nonparametric formulation of decidable counterfactual propositions in demand analysis. Suppose we observe a consumer choosing a finite number of consumption bundles $x_{i}$ at market prices $p_{i}$, i.e., $\left(p_{1}, x_{1}\right),\left(p_{2}, x_{2}\right), \ldots,\left(p_{n}, x_{n}\right)$. If the demand data is consistent with utility maximization subject to a budget constraint, i.e., satisfies GARP, the generalized axiom of revealed preference, then there exists a solution of the Afriat inequalities, $U$, that rationalizes the data, i.e., if $p_{i} \cdot x \leq p_{i} \cdot x_{i}$ then $U\left(x_{i}\right) \geq U(x)$ for $i=1,2, \ldots, n$, where $U$ is concave, continuous, monotone and nonsatiated (Afriat, 1967; Varian, 1983). Hence we may pose the following question for any two unobserved consumption bundles $\bar{x}$ and $\hat{x}$ : Will $\bar{x}$ be revealed preferred to $\hat{x}$ for every solution of the Afriat inequalities? An equivalent formulation is the counterfactual proposition: $\bar{x}$ is not revealed preferred to $\hat{x}$ for some price vector $p$ and some utility function $U$, which is a solution of the Afriat inequalities on $\left(p_{1}, x_{1}\right),\left(p_{2}, x_{2}\right), \ldots,\left(p_{n}, x_{n}\right)$.

This proposition can be expressed in terms of the solution set for the following family of polynomial inequalities: The Afriat inequalities for the augmented data set $\left(p_{1}, x_{1}\right),\left(p_{2}, x_{2}\right), \ldots,\left(p_{n}, x_{n}\right),(p, \hat{x})$ and the inequality $p \cdot \bar{x}>p \cdot \hat{x}$, where $p$ is unobserved. If these inequalities are solvable, then the stated counterfactual proposition is true. If not, then the answer to our original question is yes. Notice that $n$ of the Afriat inequalities are quadratic multivariate polynomials in the unobservables, i.e., the product of the marginal utility of income at $\hat{x}$ and the price vector $p$.

We extend the analysis of Brown and Matzkin (1996), where the Walrasian equilibrium inequalities are derived, to encompass the computation of counterfactual equilibria in Walrasian economies.

\section{Economic Models}

Brown and Matzkin (1996) characterized the Walrasian model of competitive market economies for data sets consisting of a finite number of observations on market prices, income distributions and aggregate demand. The Walrasian equilibrium inequalities, as they are called here, are defined by the Afriat inequalities for individual demand and budget constraints for each consumer; the Afriat inequalities for profit maximization over a convex aggregate technology; and the aggregation conditions that observed aggregate demand is the sum of unobserved individual demands. The Brown-Matzkin theorem states that market data is consistent with the Walrasian model if and only if the Walrasian equilibrium inequalities are solvable for the unobserved utility levels, marginal utilities of income and individual demands. Since individual demands are assumed to be unobservable, the Afriat inequalities for each consumer are quadratic multivariate polynomials in the unobservables, i.e., the prod- 
uct of the marginal utilities of income and individual demands. ${ }^{1}$

We consider an economy with $L$ commodities and $T$ consumers. Each agent has $\mathbb{R}_{+}^{L}$ as her consumption set. We restrict attention to strictly positive market prices $S=\left\{p \in \mathbb{R}_{++}^{L}: \sum_{i=1}^{L} p_{i}=1\right\}$. The Walrasian model assumes that consumers have utility functions $u_{t}: \mathbb{R}_{+}^{L} \rightarrow \mathbb{R}$, income $I_{t}$ and that aggregate demand $\bar{x}=\sum_{t=1}^{T} x_{t}$, where

$$
u_{t}\left(x_{t}\right)=\max _{\substack{p \cdot x \leq I_{t} \\ x \geq 0}} u_{t}(x)
$$

Suppose we observe a finite number $N$ of profiles of income distributions $\left\{I_{t}^{r}\right\}_{t=1}^{T}$, market prices $p^{r}$ and aggregate demand $\bar{x}^{r}$, where $r=1,2, \ldots, N$, but we do not observe the utility functions or demands of individual consumers. When are these data consistent with the Walrasian model of aggregate demand? The answer to this question is given by the following theorems of Brown and Matzkin (1996).

Theorem 1 (Theorem 2 (Brown and Matzkin)) There exist nonsatiated, continuous, strictly concave, monotone utility functions $\left\{u_{t}\right\}_{t=1}^{T}$ and $\left\{x_{t}^{r}\right\}_{t=1}^{T}$, such that $u_{t}\left(x_{t}^{r}\right)=\max _{p^{r} \cdot x \leq I_{t}^{r}} u_{t}(x)$ and $\sum_{t=1}^{T} x_{t}^{r}=\bar{x}^{r}$, where $r=1,2, \ldots, N$, if and only if $\exists$ $\left\{\hat{u}_{t}^{r}\right\},\left\{\lambda_{t}^{r}\right\}$ and $\left\{x_{t}^{r}\right\}$ for $r=1, \ldots, N ; t=1, \ldots, T$ such that

$$
\begin{gathered}
\hat{u}_{t}^{r}<\hat{u}_{t}^{s}+\lambda_{t}^{s} p^{s} \cdot\left(x_{t}^{r}-x_{t}^{s}\right) \quad(r \neq s=1, \ldots, N ; t=1, \ldots, T) \\
\lambda_{t}^{r}>0, \quad(t=1,2, \ldots T ; r=1,2, \ldots N) \\
\hat{u}_{t}^{r}>0 \text { and } x_{t}^{r} \geq 0 \quad(r=1, \ldots, N ; t=1, \ldots, T) \\
p^{r} \cdot x_{t}^{r}=I_{t}^{r} \quad(r=1, \ldots, N ; t=1, \ldots, T) \\
\sum_{t=1}^{T} x_{t}^{r}=\bar{x}^{r} \quad(r=1, \ldots, N)
\end{gathered}
$$

(1), (2) and (3) constitute the strict Afriat inequalities; (4) defines the budget constraints for each consumer; and (5) is the aggregation condition that observed aggregate demand is the sum of unobserved individual consumer demand. This family of inequalities is called here the (strict) Walrasian equilibrium inequalities. ${ }^{2}$ The observable variables in this system of inequalities are the $I_{t}^{r}, p^{r}$ and $\bar{x}^{r}$, hence this is a nonlinear family of multivariate polynomial inequalities in unobservable utility levels $\hat{u}_{t}^{r}$, marginal utilities of income $\lambda_{t}^{r}$ and individual consumer demands $x_{t}^{r}$.

The case of homothetic utilities is characterized by the following theorem of Brown and Matzkin (1996).

\footnotetext{
${ }^{1}$ The Afriat inequalities for competitive profit maximizing firms are linear given market data see Varian (1984). Hence we limit our discussion to the nonlinear Afriat inequalities for consumers.

${ }^{2}$ Brown and Matzkin call them the equilibrium inequalities, but there are other plausible notions of equilibrium in market economies.
} 
Theorem 2 (Theorem 4 (Brown and Matzkin)) There exist nonsatiated, continuous, strictly concave homothetic monotone utility functions $\left\{u_{t}\right\}_{t=1}^{T}$ and $\left\{x_{t}^{r}\right\}_{t=1}^{T}$ such that $u_{t}\left(x_{t}^{r}\right)=\max _{p^{r} \cdot x \leq I_{t}^{r}} u_{t}(x)$ and $\sum_{t=1}^{T} x_{t}^{r}=\bar{x}_{t}^{r}$, where $r=1,2, \ldots, N$ if and only if $\exists\left\{\hat{u}_{t}^{r}\right\}$ and $\left\{x_{t}^{r}\right\}$ for $r=1, \ldots, N ; t=1, \ldots, T$ such that

$$
\begin{gathered}
\hat{u}_{t}^{r}<\hat{u}_{t}^{s} \frac{p^{s} \cdot x_{t}^{r}}{p^{s} \cdot x_{t}^{s}} \quad(r \neq s=1, \ldots, N ; t=1, \ldots, T) \\
\hat{u}_{t}^{r}>0 \text { and } x_{t}^{r} \geq 0 \quad(r=1, \ldots, N ; t=1, \ldots, T) \\
p^{r} \cdot x_{t}^{r}=I_{t}^{r} \quad(r=1, \ldots, N ; t=1, \ldots, T) \\
\sum_{t=1}^{T} x_{t}^{r}=\bar{x}^{r} \quad(r=1, \ldots, N)
\end{gathered}
$$

(6) and (7) constitute the strict Afriat inequalities for homothetic utility functions.

The Brown-Matzkin analysis extends to production economies, where firms are price-taking profit maximizers. See Varian (1984) for the Afriat inequalities characterizing the behavior of firms in the Walrasian model of a market economy.

\section{Algorithms}

An algorithm for solving the Walrasian equilibrium inequalities constitutes a specification test for multiple calibration of numerical general equilibrium models, i.e., the market data is consistent with the Walrasian model if and only if the Walrasian equilibrium inequalities are solvable.

In multiple calibration, two or more years of market data together with empirical studies on demand and production functions and the general equilibrium restrictions are used to specify numerical general equilibrium models. The maintained assumption is that the market data in each year is consistent with the Walrasian model of market economies. This assumption which is crucial to the calibration approach is never tested, as noted in Dawkins et al. (2002).

The assumption of Walrasian equilibrium in the observed markets is testable, under a variety of assumptions on consumer's tastes, using the necessary and sufficient conditions stated in Theorems 1 and 2 and the market data available in multiple calibration. In particular, Theorem 2 can be used as a specification test for the numerical general equilibrium models discussed in Shoven and Whalley (1992), where it is typically assumed that utility functions are homothetic.

If we observe all the exogenous and endogenous variables, as assumed by Shoven and Whalley, then the specification test is implemented by solving the linear program, defined by (1), (2), (3), (4) and (5) for utility levels and marginal utilities of income or in the homothetic case, solving the linear program defined by (6), (7), (8), and (9) for utility levels.

If individual demands for goods and factors are not observed then the specification test is implemented using the deterministic algorithm presented here. 
Following Varian, we can extrapolate from the observed market data available in multiple calibration to unobserved market configurations. We simply augment the equilibrium inequalities defined by the observed data with additional multivariate polynomial inequalities characterizing possible but unobserved market configurations of utility levels, marginal utilities of income, individual demands, aggregate demands, income distributions and equilibrium prices. Counterfactual equilibria are defined as solutions to this augmented family of equilibrium inequalities.

In general, the Afriat inequalities in this system will be cubic multivariate polynomials because they involve the product of unobserved marginal utilities of income, the unobserved equilibrium prices and unobserved individual demands. If the observations include the market prices then the Afriat inequalities are only quadratic multivariate polynomials in the product of the unobserved marginal utility of income and individual demand. We now present the determinisitic algorithm for the quadratic case.

\subsection{Deterministic Algorithm}

The algorithm is based on the simple intuition that if one knows the order of the utility levels over the observations for each consumer, then all we have to do is to solve a linear program. We will enumerate all possible orders and solve a linear program for each order. An important point is that the number of orders is $(N !)^{T}$, where, $N$ is the number of observations and $T$ the number of agents. Hence the algorithm will run in time bounded by a function which is polynomial in the number of commodities and exponential only in $N$ and $T$. In situations involving a large number of commodities and a small $N, T$, this is very efficient. Note that trade between countries observed over a small number of observations is an example.

Consider the strict Afriat inequalities (1), (2) and (3) of Theorem 1.

Since the set of $\hat{u}_{t}^{r}$ for which there is a solution to these inqualities is an open set, we are free to add the condition: No two $\hat{u}_{t}^{r}$ are equal. This technical condition will ensure a sort of "non-degenracy". Under this condition, using the concavity of the utility function, it can be shown that (1) and (2) are equivalent to

$$
\hat{u}_{t}^{r}>\hat{u}_{t}^{s} \Rightarrow p^{s} \cdot x_{t}^{r}>p^{s} x_{t}^{s} \quad(r \neq s=1, \ldots, N ; t=1, \ldots, T) .
$$

The system (10) is not a nice system of multivariate polynomial inequalities. But now, consider a fixed consumer $t$. Suppose we fix the order of the $\left\{\hat{u}_{t}^{r}: r=1,2, \ldots N\right\}$. Then in fact, we will see that the set of feasible consumption vectors for that consumer is a polyhedron. Indeed, let $\sigma$ be a permuation of $\{1,2 \ldots N\} . \sigma$ will define the order of the $\left\{\hat{u}_{t}^{r}: r=1,2, \ldots, N\right\}$; i.e., we will have

$$
\hat{u}_{t}^{\sigma(1)}<\hat{u}_{t}^{\sigma(2)}<\cdots<\hat{u}_{t}^{\sigma(N)} .
$$

Then define $P\left(\sigma, I_{t}\right)$ to be the set of $x=\left(x_{t}^{1}, x_{t}^{2}, \ldots, x_{t}^{N}\right)$ satsifying the following:

$$
\begin{gathered}
x_{t}^{r} \in \mathbb{R}_{++}^{L} \\
p^{\sigma(s)} \cdot x_{t}^{\sigma(r)}>p^{\sigma(s)} x_{t}^{\sigma(s)} \quad N \geq r>s \geq 1
\end{gathered}
$$




$$
p^{r} \cdot x_{t}^{r}=I_{t}^{r} \quad 1 \leq r \leq N
$$

Lemma $3 P\left(\sigma, I_{t}\right)$ is precisely the set of $\left(x_{t}^{1}, x_{t}^{2}, \ldots, x_{t}^{N}\right)$ for which there exist $\lambda_{t}^{r}, \hat{u}_{t}^{r}$ satisfying (11) so that $\hat{u}_{t}^{r}, \lambda_{t}^{r}, x_{t}^{r}$ together satisfy (1), (2), (3) and (4).

Now, fix a set of $T$ permutations $-\sigma_{1}, \sigma_{2}, \ldots, \sigma_{T}$, one for each consumer. We can then write a linear system of inequalities for $P\left(\sigma_{t}, I_{t}\right)$ for $t=1,2, \ldots, T$ and the consumption total (5). If this system is feasible, then there is a feasible solution with the utilities in the given order. Clearly, if all such $(N !)^{T}$ systems are infeasible, then there is no rationalization.

Remark If for a particular consumer $t$,

$$
\frac{p^{1}}{I_{t}^{1}} \geq \frac{p^{2}}{I_{t}^{2}} \geq \cdots \geq \frac{p^{N}}{I_{t}^{N}},
$$

then, by Lemma 1 of Brown-Shannon (2004), we may assume that the $u_{t}^{r}$ are nondecreasing. For such a consumer, only one order needs to be considered. Note that the above condition says that in a sense, the consumer's income outpaces inflation on each and every good.

A more challenging problem is the computation of counterfactual equilibria. Fortunately, a common restriction in applied general equilibrium analysis is the assumption that consumers are maximizing homothetic utility functions subject to their budget constraints and firms have homothetic production functions. A discussion of the Afriat inequalities for cost minimization and profit maximization for firms with homothetic production functions can be found in Varian (1984). Afriat (1981) and subsequently Varian (1983) derived a family of inequalities in terms of utility levels, market prices and incomes that characterize consumer's demands if utility functions are homothetic. We shall refer to these inequalities as the homothetic Afriat inequalities.

Following Shoven and Whalley (1992), see page 107, we assume that we observe all the exogenous and endogenous market variables in the benchmark equilibrium data sets, used in the calibration exercise. As an example, suppose there is only one benchmark data set, then the (strict) homothetic Afriat inequalities for each consumer are of the form: ${ }^{3}$

$$
\begin{array}{lll}
U^{1}<\lambda^{2} p^{2} \cdot x^{1} & \text { and } & U^{2}<\lambda^{1} p^{1} \cdot x^{2} \\
U^{1}=\lambda^{1} I^{1} & & U^{2}=\lambda^{2} I^{2}
\end{array}
$$

where we observe $p^{1}, x^{1}$ and $I^{1}$. Given $\lambda^{1}$ and $\lambda^{2}$ we again have a linear system of inequalities in the unobserved $U^{1}, U^{2}, x^{2}, p^{2}$ and $I^{2}$. A similar set of inequalities can be derived for cost minimizing or profit maximizing firms with production functions that are homogenous of degree one.

\footnotetext{
${ }^{3}$ Here we assume utility functions are homogenous of degree one.
} 


\subsection{VC algorithm}

For the general case (with no assumptions on homotheticity or knowledge of all price vectors), we propose the "VC algorithm"; here we give a brief description of this with details to follow. First, let $y$ be a vector consisting of marginal utilities of income and utility levels of each consumer in each observation (so $y \in \mathbb{R}^{k}$, where $k=2 N T$ ). Let $x$ be a vector comprising the consumption vectors for each consumer in each observation (so $x \in \mathbb{R}^{n}$, where $n=N T L$ ). Finally, let $z$ be a vector comprising the unknown price vector in the case of the counterfactual proposition. Then our inequalities are multivariate polynomials in the variables $(x, y, z)$; but they are multi-linear in the $x, y, z$. We would like to find a solution $(x, y, z)$ in case one exists. Suppose now that the proportion of all $(x, y, z)$ which are solutions is some $\delta>0$. A simple algorithm for finding a solution would be to pick at random many $(x, y, z)$ triples and check if any one of these solve the inequalities. The number of trials we need is of the form $1 / \delta$, In general, $\delta$ may be very small (even if positive) and we have to do many trials. Instead consider another parameter $-\varepsilon$ defined by

$$
\varepsilon=\operatorname{Max}_{y} \text { (proportion of }(x, z) \text { such that }(x, y, z) \text { solve the inequalities.) }
$$

Clearly, $\varepsilon$ is always at least $\delta$, but it is typically much larger. Using the concept of Vapnik-Chervonenkis (VC) dimension, we will give an algorithm which makes $O(d / \varepsilon)$ trials, where $d$ is the $\mathrm{VC}$ dimension of the $\mathrm{VC}$ class of sets defined by our inequalities. We elaborate on this later, but remark here that $d$ is $O(N T(\log N T L))$. Thus when at least one $y$ admits a not too small proportion of $(x, z)$ as solutions, this algorithm will be efficient.

To develop the algorithm, we construct an $\varepsilon$-net - a small set $S$ of $(x, z)$ such that with high probability, every set in the $(x, z)$ space containing at least $\varepsilon$ proportion of the $(x, z)$ 's has an element of $S$. This $\varepsilon$-net is derived from the VC-property of the Walrasian equilibrium inequalities. That is, Laskowski (1992) has shown that any finite family of multivariate polynomial inequalities in unknowns $(x, y, z)$, defines a VC-class of sets. As is well known in computational geometry, VC-classes imply the existence of "small" $\varepsilon$-nets.

In our setting, at each point in the $\varepsilon$-net, the Walrasian inequalities define a linear program in the $y$ 's, since the Walrasian equilibrium inequalities are a system of multilinear polynomial inequalities. One of these linear programs will yield a solution to our system. Of course, $\varepsilon$ as defined above is not known in advance. We just try first 1 as a value for $\varepsilon$ and if this fails, we half it and so on until we either have success or the value tried is small enough, whence we may stop and assert with high confidence that for every $y$, the proportion of $(x, z)$ which solve the inequalities is very small. (This includes the case of an empty set of solutions.)

More generally our "VC-algorithm" is a decision method for any finite family of multilinear polynomial inequalities, e.g., testable restrictions of strategic games see Carvajal et al. (2004). This algorithm is polynomial in the number of variables and $1 / \varepsilon$. Now the details.

A collection $\mathcal{C}$ of subsets of some space $\chi$ picks out a certain subset $E$ of a finite set $\left\{x_{1}, x_{2}, \ldots, x_{n}\right\} \subset \chi$ if $E=\left\{x_{1}, \ldots, x_{n}\right\} \cap A$ for some $A \in \mathcal{C}$. $\mathcal{C}$ is said to shatter 
$\left\{x_{1}, \ldots, x_{n}\right\}$ is $\mathcal{C}$ picks out each of its $2^{n}$ subsets. The VC-dimension of $\mathcal{C}$, denoted $V(\mathcal{C})$ is the smallest $n$ for which no set of size $n$ is shattered by $\mathcal{C}$. A collection $\mathcal{C}$ of measurable sets is called a VC-class if its dimension, $V(\mathcal{C})$, is finite.

Let $y$ be a vector consisting of marginal utilities of income and utility levels of each consumer in each observation (so $y \in \mathbb{R}^{k}$, where $k=2 N T$ ). Let $x$ be a vector comprising the consumption vectors for each consumer in each observation (so $x \in \mathbb{R}^{n}$, where $n=N T L$ ). Finally, let $z$ be a vector comprising the unknown price vector in the case of the counterfactual proposition (so $z \in \mathbb{R}^{L}$ ). The Walrasian equilibrium inequalities (1) through (5) define a Boolean formula $\Phi(x, y, z)$ containing $s \in O\left(N^{2} T+N T L\right)$ atomic predicates where each predicate is a polynomial equality or inequality over $n+k+L$ variables $-x, y, z$. Here $\Phi$ is simply the conjunction of the inequalities (1) through (5). For any fixed $y$, let $F_{y} \subseteq \mathbb{R}^{n+L}$ be the set of $(x, z)$ such that $\Phi(x, y, z)$ is satisfied then this is a VC-class of sets by Laskowski's Theorem. Let $\varepsilon=\operatorname{Max}_{y}$ (proportion of $(x, z)$ such that $(x, y, z)$ solve the inequalities.) Note that if the set of solutions has measure 0 (or in particular is empty), then $\varepsilon=0$.

Theorem 4 (Goldberg-Jerrum (1995)) The family $\left\{F_{y}: y \in \mathbb{R}^{k}\right\}$ has $V C$-dimension at most $2 k \log _{2}(100 s)$.

The next proposition is a result from Blumer et.al. (1989).

Theorem 5 If $\mathcal{F}$ is a $V C$-class of subsets of $X$ with $V C$-dimension $d$, and $\varepsilon, \delta$ are positive reals and $m \geq\left(8 d / \varepsilon \delta^{2}\right) \log _{2}(13 / \varepsilon)$, then a random ${ }^{4}$ subset $\left\{x_{1}, x_{2}, \ldots, x_{m}\right\}$ of $X$ (called a $\varepsilon$-net) satisfies the following with probabilty at least $1-\delta$ :

$$
S \cap\left\{x_{1}, \ldots, x_{m}\right\} \neq \phi \quad \forall S \in \mathcal{F} \text { with } \mu(S) \geq \varepsilon .
$$

For fixed $\bar{x}, \bar{z}$, the Walrasian equilibrium inequalities define a linear program over $y \in \mathbb{R}_{+}^{k}$. Hence each point in the $\varepsilon$-net defines a linear program in the $y$ 's. Now, the random algorithm is clear: we pick a random set of $m(x, z)$ pairs, where $\varepsilon$ is defined above and solve a linear program for each. For the correct $\varepsilon$, we will succeed with high probability in finding a solution. We do not know the $\varepsilon$ a priori, but we use a standard trick of starting with $\varepsilon=1$ and halving it each time until we have success or we stop with a small upper bound on $\varepsilon$.

A preliminary version of the VC-algorithm was introduced in Brown and Kannan (2003). But the bound here on the VC dimension using Theorem 5 and hence the running time are substantially better than those derived in that report.

Acknowledgments This revision has benefited from our reading of Kubler's (2004) unpublished manuscript. We thank him, Glena Ames and Louise Danishevsky for their assistance.

\footnotetext{
${ }^{4}$ picked in indepedent, uniform trials
} 


\section{References}

Afriat, S. (1967). "The Construction of a Utility Function from Expenditure Data," International Economic Review, 66-77.

(1981). "On the Constructability of Consistent Price Indices between Several Periods Simultaneously." In A. Deaton (ed.), Essays in Applied Demand Analysis. Cambridge: Cambridge University Press.

Blumer, A., A. Ehrenfeucht, D. Haussler and M. Warmuth (1989). "Learnability and the Vapnik-Cerovenkis Dimension," Journal of the AMC, 36, 929-965.

Brown, D. and R. Kannan (2003). "Indeterminacy, Nonparametric Calibration and Counterfactual Equilibria," Cowles Foundation Discussion Paper No. 1426, Yale University.

Brown, D. and R. Matzkin (1996). "Testable Restrictions on the Equilibrium Manifold," Econometrica, 64, 1249-1262.

Brown, D. and C. Shannon (2000). "Uniqueness, Stability, and Comparative Statics in Rationalizable Walrasian Markets," Econometrica, 68, 1529-1539.

Carvajal, A., et al. (2004). "Equilibrium Behavior in Markets and Games: Testable Restrictions and Identification," Journal of Mathematical Economics, 40: 1-40.

Dawkins, C., T.N. Srinivasan and J. Whalley (2000). "Calibration." In J.J. Heckman and E. Leamer (eds.), Handbook of Econometrics, Vol. 5. New York: Elsevier.

Goldberg, P. and M. Jerrum (1995). "Bounding the Vapnik-Čerovenkis Dimension of Concept Classes Parameterized by Real Numbers," Machine Learning, 18, $131-148$.

Kubler, F. (2004). "Approximate Generalizations and Applied Equilibrium Analysis," unpublished manuscript, Department of Economics, University of Mannheim.

Laskowski, C. (1992). "Vapnik-Čerovenkis Classes of Definable Sets," Journal of the London Mathematical Society, 294, 231-251.

Shoven, J. and J. Whalley (1992). Applying General Equilibrium. Cambridge: Cambridge University Press.

Varian, H. (1982). "The Nonparametric Approach to Demand Analysis," Econometrica, 50, 945-973.

(1983). "Non-parametric Tests of Consumer Behavior," Review of Economic Studies, 99-110.

(1984). "Nonparametric Approach to Production Analysis," Econometrica, $52,579-597$. 